\title{
Quantum criticality of a one-dimensional Bose-Fermi mixture
}

\author{
Xiangguo Yin, ${ }^{1}$ Xi-Wen Guan, ${ }^{2, *}$ Yunbo Zhang, ${ }^{3}$ and Shu Chen ${ }^{1, \dagger}$ \\ ${ }^{1}$ Beijing National Laboratory for Condensed Matter Physics, Institute of Physics, Chinese Academy of Sciences, Beijing 100190, China \\ ${ }^{2}$ Department of Theoretical Physics, Research School of Physics and Engineering, Australian National University, \\ Canberra ACT 0200, Australia \\ ${ }^{3}$ Department of Physics and Institute of Theoretical Physics, Shanxi University, Taiyuan 030006, China
}

(Received 28 November 2011; published 4 January 2012)

\begin{abstract}
The one-dimensional interacting Bose-Fermi mixtures, exhibiting quantum phase transitions at zero temperature, are particularly valuable for the study of quantum critical phenomena. In the present paper, we analytically study the quantum phase diagram, equation of state, and quantum criticality of the Bose-Fermi mixture using the thermodynamic Bethe ansatz equations. We show that thermodynamical properties display universal scaling behavior at quantum criticality. Furthermore, quantum criticality of the Bose-Fermi mixture in a harmonic trap is also studied within the local-density approximation. We thus demonstrate that the phase diagram and critical properties of the bulk system provide insight into understanding the universal features of many-body critical phenomena.
\end{abstract}

DOI: 10.1103/PhysRevA.85.013608

PACS number(s): 03.75.Mn, 03.75.Hh, 02.30.Ik, 05.30.Rt

\section{INTRODUCTION}

Mixtures of ultracold bosonic and fermionic atoms have attracted intensive studies both experimentally and theoretically [1-3]. By loading cold atoms in one-dimensional (1D) waveguides and tuning the effective interactions by Feshbach resonance, it is possible to simulate striking quantum many-body phenomena in 1D strongly correlated systems in the whole regime of interaction strength [4-6]. The exquisite tunability with ultracold atoms confined to low dimensions has provided unprecedented opportunities for investigating and testing the theory of exactly solvable many-body systems [7-11]. These include remarkable experimental progress in the realization of Tonks-Girardeau gas [7,8], super-Tonks-Girardeau gas [9], Yang-Yang thermodynamics for ultracold Bose gas of ${ }^{87} \mathrm{Rb}$ [10], and the exotic density profiles of the attractive Fermi gas in a harmonic trap [11]. The current experimental progress is capable of simulating 1D Bose-Fermi mixtures.

Recently, various theoretical methods have been used to study quantum phases and correlations of the 1D Bose-Fermi mixtures, such as the mean-field approach [12] and TomonagaLuttinger liquid (TLL) theory [13,14]. The 1D Bose-Fermi mixture with equal masses of bosons and fermions, and with the same strength of $\delta$-function interaction between bosonboson and boson-fermion, is exactly solvable [15]. This model has attracted renewed interest [16-19] due to the experimental progress with cold atomic systems.

Particular theoretical interest has been paid to the groundstate properties at zero temperature [16-18]. However, there are very few studies on the thermodynamics and quantum critical phenomena of the model. In review of the realistic cold atomic systems trapped in external potentials at finite temperatures, it is significantly important to understand how to unambiguously determine the zero-temperature phase diagram from the knowledge of finite-temperature quantities of trapped gases. In the 1D mixture of quantum gases, true

\footnotetext{
*xwe105@rsphysse.anu.edu.au

${ }^{\dagger}$ schen@aphy.iphy.ac.cn
}

quantum phase transitions occur as the driving parameters vary across the phase boundaries at zero temperature, such as chemical potential, magnetic field and densities, etc. In particular, 1D quantum critical phenomena associated with quantum phase transitions at zero temperature give the physical origin of quantum criticality [20,21] and provide an insight into the understanding of the universal scaling behavior of thermodynamical properties in quantum critical regimes [22-25]. By using the universal scaling functions, it has been demonstrated that the zero-temperature phase diagrams of various systems can be mapped out from the finite-temperature density profiles [22,24,26]. Most recently, the high-resolution imaging techniques have allowed measurement of the density profiles and density fluctuations of the trapped atomic gases very precisely [27-31], and thus provide essential tools to study quantum phase transitions and quantum criticality.

In general, quantum fluctuations are strongly coupled with thermal fluctuations in the quantum critical regime. Therefore, quantum criticality is among the most challenging of problems in condensed-matter physics. In order to extract correct universal scaling functions, which control proper thermal and quantum fluctuations at quantum criticality, a high precision of the finite-temperature thermodynamics is desirable. Usually, access to the thermodynamic properties of integrable models at finite temperatures is notoriously difficult and presents a formidable challenge in theoretical and mathematical physics. In the present paper, we analytically determine the zero-temperature phase diagram of the integrable Bose-Fermi mixture. We further derive the equation of state and explore the universal scaling behavior of thermodynamical properties at quantum criticality using the thermodynamical Bethe ansatz (TBA) equations. Using the exact analytical result obtained, we also demonstrate that the zero-temperature phase diagram and quantum criticality can be mapped out from finite-temperature density profiles of the trapped gas within the local-density approximation.

The paper is organized as follows. In Sec. II, we present the TBA equations for the model and analytically determine the phase diagram of the Bose-Fermi mixture at zero temperature. In Sec. III, we derive the equation of state and explore the 
universal scaling behavior of the density and compressibility near the critical points. In Sec. IV, the quantum criticality of the gas in a harmonic trap is studied within the local-density approximation. A conclusion is presented in Sec. V.

\section{MODEL AND PHASE DIAGRAM AT ZERO TEMPERATURE}

We consider a 1D interacting Bose-Fermi mixture described by the Hamiltonian

$$
\begin{aligned}
\hat{H}= & \int_{0}^{L} d x\left(\frac{\hbar^{2}}{2 m_{b}} \partial_{x} \Psi_{b}^{\dagger} \partial_{x} \Psi_{b}+\frac{\hbar^{2}}{2 m_{f}} \partial_{x} \Psi_{f}^{\dagger} \partial_{x} \Psi_{f}\right. \\
& +\frac{g_{b b}}{2} \Psi_{b}^{\dagger} \Psi_{b}^{\dagger} \Psi_{b} \Psi_{b}+g_{b f} \Psi_{b}^{\dagger} \Psi_{f}^{\dagger} \Psi_{f} \Psi_{b} \\
& \left.-\mu_{f} \Psi_{f}^{\dagger} \Psi_{f}-\mu_{b} \Psi_{b}^{\dagger} \Psi_{b}\right)
\end{aligned}
$$

where $\Psi_{b}$ and $\Psi_{f}$ are boson and fermion field operators, $m_{b}$ and $m_{f}$ are the masses, $\mu_{b}$ and $\mu_{f}$ are chemical potentials of bosons and fermions, and $g_{b b}$ and $g_{b f}$ are boson-boson and boson-fermion interaction strengths, respectively. Here we consider the fully polarized fermions, therefore, the Pauli principle excludes their $s$-wave interaction $\left(g_{f f}=0\right)$. This model is exactly solvable $[15,16]$ for equal masses and equal boson-boson and boson-fermion interaction strengths, i.e., $m_{b}=m_{f}=m$ and $g_{b b}=g_{b f}=g$. For our convenience, we assume $\hbar=2 m=1$. The first quantization form of the exactly solvable Hamiltonian (1) can be written as

$$
\hat{H}=-\sum_{i=1}^{N} \frac{\partial^{2}}{\partial x_{i}^{2}}+2 c \sum_{i<j} \delta\left(x_{i}-x_{j}\right)-\mu N-\frac{H}{2}\left(N_{f}-N_{b}\right),
$$

with $c=m g / \hbar^{2}$. Here the particle number $N=N_{b}+N_{f}$, with $N_{b}$ bosons and $N_{f}$ fermions. The chemical potential $\mu$ and the effective magnetic field $H$ are defined as $\mu=\left(\mu_{f}+\mu_{b}\right) / 2$ and $H=\mu_{f}-\mu_{b}$. The many-body wave function is supposed to be symmetric with respect to indices $i=\left\{1,2, \ldots, N_{b}\right\}$ (bosons) and antisymmetric with respect to $\left\{N_{b}+1, N_{b}+\right.$ $\left.2, \ldots, N_{f}\right\}$ (fermions). Thus, the $N$-body wave function can be written as $N ! \times N !$ superpositions of individual plane waves associated with $N$ quasimomenta $k_{i}$, with $i=1, \ldots, N$ by means of the Bethe ansatz $[15,16]$.

The spectrum of the system is given by $E=\sum_{i=1}^{N} k_{i}^{2}$, where the quasimomenta $k_{i}$ is subject to the so-called Bethe ansatz equations (BAEs) $[15,16]$. In thermodynamic limits, $N \rightarrow$ $\infty, L \rightarrow \infty$, and $N / L$ is finite, and at finite temperatures, the equilibrium states become degenerate. Yang and Yang [32] showed that true physical states in integrable systems can be determined from the minimization conditions of Gibbs free energy subject to the Bethe ansatz equations. Accordingly, in the thermodynamics limit with $N_{b} / L$ and $N_{f} / L$ fixed, minimization of the Gibbs free energy gives rise to the following nonlinear integral equations [19], i.e., the TBA equations for the integrable Bose-Fermi mixture $\left(k_{B}=1\right)$ :

$$
\begin{aligned}
\epsilon(k)= & k^{2}-\mu_{f}-T \int_{-\infty}^{\infty} a_{1}(\Lambda-k) \\
& \times \ln \{1+\exp [-\varphi(\Lambda) / T]\} d \Lambda,
\end{aligned}
$$

$$
\begin{aligned}
\varphi(\Lambda)= & \mu_{f}-\mu_{b}-T \int_{-\infty}^{\infty} a_{1}(k-\Lambda) \\
& \times \ln \{1+\exp [-\epsilon(k) / T]\} d k,
\end{aligned}
$$

where $a_{\ell}(x)=\frac{1}{2 \pi} \frac{\ell c}{(\ell c)^{2} / 4+x^{2}}$. For fixed temperature $T$ and chemical potential $\mu_{f}, \mu_{b}$, the pressure is given by

$$
p=\frac{T}{2 \pi} \int_{-\infty}^{\infty} \ln \left\{1+\exp \left[-\frac{\epsilon(k)}{T}\right]\right\} d k .
$$

The particle density and compressibility for fermions and bosons, and the entropy per length, can be obtained from

$$
\begin{gathered}
n_{i} \equiv N_{i} / L=\partial p / \partial \mu_{i}, \quad i=f, b, \\
\kappa_{i} \equiv \partial n_{i} / \partial \mu, \quad i=f, b, \\
S / L \equiv \partial p / \partial T .
\end{gathered}
$$

The phase diagram of the Bose-Fermi mixture can be analytically determined from the TBA equations (3) in the zero-temperature limit. As shown in Fig. 1, the phase diagram consists of three quantum phases: pure bosons, pure fermions, and the mixture of bosons and fermions except the vacuum, which are separated by four boundary lines with condition $n_{f}=0$ or $n_{b}=0$. In order to obtain the full phase diagram of the Bose-Fermi mixture, we introduce two sets of the TBA equations with different reference states [33]. The TBA equations based on the fermionic reference state determine the two boundary lines for $H>0$, whereas the TBA equations based on the bosonic reference state determine the two boundary lines for $H \leqslant 0$.

In the limit $T \rightarrow 0$, the TBA equations (3) based on the fermionic reference state reduce to

$$
\begin{gathered}
\epsilon(k)=k^{2}-\mu_{f}+\int_{-\Lambda_{F}}^{\Lambda_{F}} a_{1}(\Lambda-k) \varphi^{-}(\Lambda) d \Lambda, \\
\varphi(\Lambda)=\mu_{f}-\mu_{b}+\int_{-k_{F}}^{k_{F}} a_{1}(k-\Lambda) \epsilon^{-}(k) d k,
\end{gathered}
$$

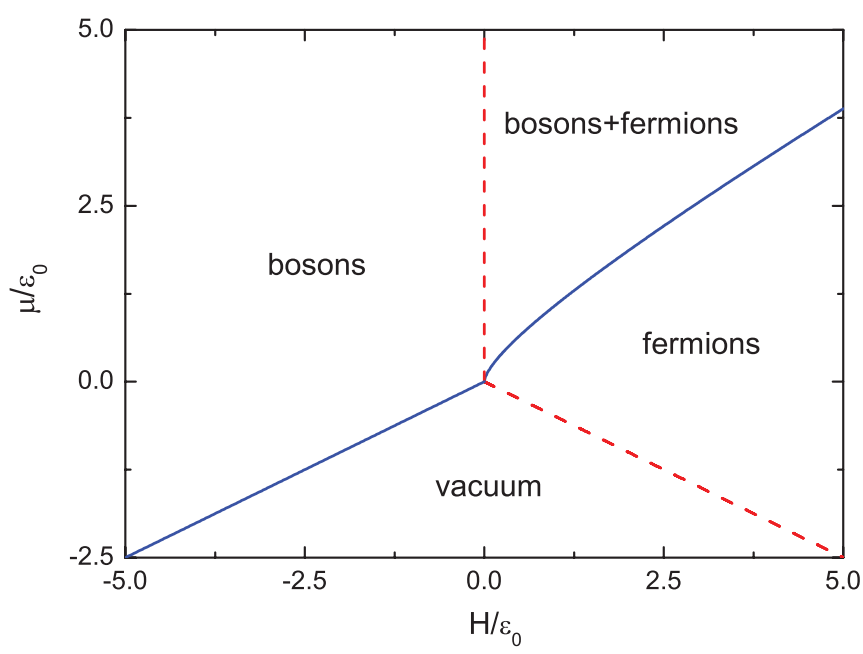

FIG. 1. (Color online) Phase diagram in the $\mu-H$ plane. Three distinguished phases result from varying the chemical potential and magnetic field, i.e., pure boson phase for $H<0$ and $\mu>H / 2$, pure fermion phase below the phase boundary (12) in the region $\mu>$ $-H / 2$, and the mixture of bosons and fermions above the phase boundary (12) in the region $H>0$. 
where the dressed energies $\epsilon^{-}(k)$ and $\varphi^{-}(\Lambda)$ correspond to the occupied states for $k \in\left[-k_{F}, k_{F}\right]$ and $\Lambda \in\left[-\Lambda_{F}, \Lambda_{F}\right]$, respectively. The positive parts of the dressed energies $\epsilon(k)$ and $\varphi(\Lambda)$ correspond to the unoccupied states. The integration boundaries $k_{F}$ and $\Lambda_{F}$ characterize the Fermi points with the conditions $\epsilon^{-}\left( \pm k_{F}\right)=\varphi^{-}\left( \pm \Lambda_{F}\right)=0$. The pressure is given by

$$
p=-\frac{1}{2 \pi} \int_{-k_{F}}^{k_{F}} \epsilon^{-}(k) d k
$$

at zero temperature. We calculate the particle densities through the relations (5). Nevertheless, the boundary lines, which correspond to $n_{f}=0$ or $n_{b}=0$, can be determined by analyzing the dressed energy at the point $k_{F}=0$ or $\Lambda_{F}=0$.

For a pure fermion state, $\varphi(\Lambda)$ is gapful, i.e., $\varphi(\Lambda)>0$. Thus, the TBA equations (8) reduce to the free fermion potential $\epsilon(k)=k^{2}-\mu_{f}$. The phase boundaries for the phase transitions from vacuum into the pure fermion state, and from the pure fermion state into the mixture of bosons and fermions, are determined by

$$
\begin{aligned}
& \epsilon^{-}(0) \leqslant 0, \\
& \varphi^{-}(0) \leqslant 0,
\end{aligned}
$$

respectively. From the condition (10), we obtain the phase boundary for pure fermions $\mu_{f} \geqslant 0$, or, equivalently, $\mu \geqslant$ $-H / 2$.

From the conditions (11) and $\epsilon(k)=k^{2}-\mu_{f}$, we obtain the phase boundary

$$
\tilde{H} \leqslant \frac{1}{2 \pi}\left[\left(4 \tilde{\mu}_{f}+1\right) \arctan \sqrt{4 \tilde{\mu}_{f}}-\sqrt{4 \tilde{\mu}_{f}}\right]
$$

for the coexistence of bosons and fermions. Here we used the dimensionless units $\tilde{H} \equiv H / \varepsilon_{0}$ and $\tilde{\mu}_{f} \equiv \mu_{f} / \varepsilon_{0}$ with $\varepsilon_{0}=c^{2}$. For the strong-coupling regime, i.e., $\mu_{f} \ll \varepsilon_{0}$ or $H \ll \varepsilon_{0}$, we find that phase boundary condition (12) reduces to

$$
\tilde{\mu} \geqslant \frac{1}{4}\left\{(3 \pi \tilde{H})^{2 / 3}\left[1+\frac{2}{15}(3 \pi \tilde{H})^{2 / 3}\right]-2 \tilde{H}\right\},
$$

with $\tilde{\mu} \equiv \mu / \varepsilon_{0}$. For the weak-coupling regime, i.e., $\mu_{f} \ll \varepsilon_{0}$ or $H \gg \varepsilon_{0}$, the phase boundary condition becomes

$$
\tilde{\mu} \geqslant \frac{\tilde{H}}{2}+\frac{2}{\pi} \sqrt{\tilde{H}}-\frac{1}{4}\left(1-\frac{8}{\pi^{2}}\right) .
$$

On the other hand, at zero temperature, the TBA equations with the Bose state as the reference state are given by [33]

$$
\begin{aligned}
\epsilon(k)= & k^{2}-\mu_{b}+\int_{-k_{F}}^{k_{F}} a_{2}\left(k-k^{\prime}\right) \epsilon^{-}\left(k^{\prime}\right) d k^{\prime} \\
& +\left(\int_{-\infty}^{-\Lambda_{F}}+\int_{\Lambda_{F}}^{\infty}\right) a_{1}(k-\Lambda) \varphi^{-}(\Lambda) d \Lambda, \\
\varphi(\Lambda)= & \mu_{b}-\mu_{f}-\int_{-k_{F}}^{k_{F}} a_{1}(k-\Lambda) \epsilon^{-}(k) d k,
\end{aligned}
$$

with the Fermi points $\epsilon^{-}\left( \pm k_{F}\right)=\varphi^{-}\left( \pm \Lambda_{F}\right)=0$. Similarly, the dressed energies $\epsilon^{-}(k)$ and $\varphi^{-}(\Lambda)$ correspond to the occupied states for $k \in\left[-k_{F}, k_{F}\right]$ and $\Lambda \in\left[-\Lambda_{F}, \Lambda_{F}\right]$, respectively. We see that for the Bose reference state, the fully polarized fermions provide a ferromagnetic ordering at the ground state. If $H<0$, i.e., $\mu_{f}<\mu_{b}$, then the dressed energy
$\varphi(\Lambda)$ is greater than zero. The dressed energy $\varphi$ is gapful. Thus, the ground state is a pure boson state. Therefore, for $H<0$, the TBA equations (15) reduce to Yang-Yang thermodynamics equations for the Lieb-Liniger Bose gas [32],

$$
\epsilon(k)=k^{2}-\mu_{b}+\int_{-k_{F}}^{k_{F}} a_{2}\left(k-k^{\prime}\right) \epsilon^{-}\left(k^{\prime}\right) d k^{\prime},
$$

from which we easily determine the phase boundary for the phase transition from vacuum into the pure boson state, i.e., $\mu \geqslant H / 2$; see Fig. 1 .

\section{EQNARRAY OF STATE AND UNIVERSAL SCALINGS}

Recent experiments on quantum criticality of ultracold atoms [28,31] and a theoretical scheme of mapping out quantum criticality of cold atoms [22-25] open the possibility to explore such universal behavior in low-dimensional, multicomponent, interacting Fermi and Bose gases. As the temperature is tuned over the crossover temperatures, the scaling functions of thermodynamical properties give rise to universal behavior, which entirely depends on the symmetry of the excitation spectrum and dimensionality of the system. This gives a promising way to explore the hidden symmetry of these models, for example, the quantum Ising model with transverse file displays emergent $E_{8}$ symmetry [35]. In the critical regime, the thermodynamic functions of the homogeneous gas can be cast into some universal scaling forms [20,21]. For example, the density and compressibility near the critical point $\mu=\mu_{c}$ can be written as

$$
\begin{aligned}
& n(T, \mu)=n_{0}+T^{\frac{d}{z}+1-\frac{1}{v z}} \mathcal{G}\left(\frac{\mu-\mu_{c}}{T^{\frac{1}{v z}}}\right), \\
& \kappa(T, \mu)=\kappa_{0}+T^{\frac{d}{z}+1-\frac{2}{v z}} \mathcal{F}\left(\frac{\mu-\mu_{c}}{T^{\frac{1}{v z}}}\right) .
\end{aligned}
$$

Here, $n_{0}\left(\kappa_{0}\right)$ is the regular part of the density (compressibility) induced from the background, $\mathcal{G}(\mathcal{F})$ is a universal scaling function describing the singular part of the density (compressibility) near the critical point $\mu_{c}, d$ is the dimensionality of the system, $z$ is the dynamical critical exponent, and $v$ is the correlation length exponent. From the above relation, the dimensionless universal scaling functions $\mathcal{G}\left(\frac{\mu-\mu_{c}}{T^{v z}}\right)$ and $\mathcal{F}\left(\frac{\mu-\mu_{c}}{T^{v z}}\right)$ display universal scaling behavior near the critical point of $\mu=\mu_{c}$, i.e., the density (compressibility) curves with a subtraction of the background density (compressibility) intersect at the critical point for different temperatures. This feature can be used to detect the phase boundaries at zero temperature from finite-temperature density profiles of the trapped gas.

Before discussing the universal scaling behavior of the Bose-Fermi mixture, we will discuss quantum criticality of several simple examples. The simplest example is the 1D ideal Fermi gas. The ideal Fermi gas obeys the Fermi-Dirac distribution; it is easy to derive the density distribution for free fermions $n_{f}(T, \mu)=-\sqrt{T} /(2 \sqrt{\pi}) \mathrm{Li}_{1 / 2}\left(-\exp \left(\frac{\mu_{f}}{T}\right)\right)$, where $\operatorname{Li}_{n}(x)=\sum_{l=1}^{\infty} x^{l} / l^{n}$ is the standard polylogarithmic function. In comparison with the universal scaling given by Eq. (16), the critical exponent $z=2$ and the correlation length exponent $v=1 / 2$ with the dimensionality $d=1$ can be read off of the universal scaling form. Here the scaling function is 
$\mathcal{G}(x)=-1 /(2 \sqrt{\pi}) \mathrm{Li}_{1 / 2}(-\exp (x))$. There is no background density for the vacuum-Fermi-gas transition, i.e., $n_{0}=0$, where the critical point $\mu_{c}=0$. It was also shown that $d=1$, $z=2$, and $v=1 / 2$ for the vacuum into the TLL phase transition in the 1D hard-core bosons [22], the 1D attractive Fermi gas [24], and the 1D interacting Bose gas with strongly repulsive interactions [25].

In order to investigate the quantum critical behavior in the vicinity of the phase boundary between the phase of a mixture of bosons and fermions and the phase of the fully polarized fermions, we will derive the equation of state from the TBA equations (3). For the strongly interacting regime, i.e., $c \gg 1$, or $T / \varepsilon_{0} \gg 1$, we can rewrite the TBA equations (3) as

$$
\begin{aligned}
& \epsilon(k) \approx k^{2} / \beta-A, \\
& \varphi(\Lambda)=\mu_{f}-\mu_{b}-\frac{4 p c}{c^{2}+4 \Lambda^{2}}+\frac{4 c\left(4 c^{2}-48 \Lambda^{2}\right) p_{2}}{\left(c^{2}+4 \Lambda^{2}\right)^{3}},
\end{aligned}
$$

where

$$
\begin{aligned}
A & \approx \mu_{f}+T \int_{-\infty}^{\infty} a_{1}(\Lambda) \ln \left\{1+\exp \left[-\frac{\varphi(\Lambda)}{T}\right]\right\} d \Lambda, \\
\beta & =1-\frac{2 T c}{\pi} \int_{-\infty}^{\infty} \frac{4 c^{2}-48 \Lambda^{2}}{\left(c^{2}+4 \Lambda^{2}\right)^{3}} \ln \left[1+e^{-\frac{\varphi(\Lambda)}{T}}\right] d \Lambda, \\
p_{2} & =-\frac{\beta T^{2}}{4 \sqrt{\pi}} \operatorname{Li}_{\frac{5}{2}}\left(-e^{\frac{A}{T}}\right) .
\end{aligned}
$$

With the help of these relations, the pressure (4) can be calculated in a straightforward way as

$$
p=-\sqrt{\frac{\beta}{4 \pi}} T^{\frac{3}{2}} \operatorname{Li}_{\frac{3}{2}}\left(-e^{\frac{A}{T}}\right)
$$

that serves as the equation of state of the model with strong repulsion. The thermodynamical properties can be obtained from the usual thermodynamical relations. This analytical equation of state (20) essentially covers the universal TLL thermodynamics and encodes the critical exponents in the critical regimes.

At very low temperatures, i.e., $T \ll \varepsilon_{0}$, the thermodynamics of the model is governed by the TLL physics associated with a linear dispersion. In the mixed phase of bosons and fermions, the low-energy physics belongs to a universality class of a two-component TLL $[17,18]$. In this low-temperature limit, we further calculate the pressure

$$
p \approx \frac{2 A^{3 / 2}}{3 \pi}\left(1+\frac{\pi^{2}}{8} \frac{T^{2}}{A^{2}}\right)
$$

where

$$
\begin{aligned}
A \approx \mu & +\frac{H}{2}-\frac{4}{\pi}\left(\frac{H}{2}-\frac{p}{c}\right) \tan ^{-1} \frac{2 \Lambda_{0}}{c} \\
+ & \frac{2 H \Lambda_{0}}{\pi c}+\frac{c \pi T^{2}}{12 H \Lambda_{0}},
\end{aligned}
$$

with $\Lambda_{0}=c \sqrt{\frac{p}{H c}-\frac{1}{4}}$. After a lengthy algebra, we find a universal leading order of temperature corrections to the free energy,

$$
F=E_{0}-\frac{\pi C T^{2}}{6}\left(\frac{1}{v_{b}}+\frac{1}{v_{f}}\right)
$$

where the two velocities in the strongly repulsive regime are given by

$$
\begin{aligned}
& v_{s}=\frac{4 \pi^{2} n}{3 \gamma} \sin (\pi \alpha), \\
& v_{f}=2 \pi n\left\{1-\frac{4}{\gamma}[\pi \alpha+\sin (\pi \alpha)]\right\} .
\end{aligned}
$$

The parameter $\alpha$ is determined by the relation $\alpha \approx n_{b} / n$ for a small $H \ll 1$. The ground-state energy is given by

$$
E=\frac{1}{3} n^{3} \pi^{2}\left\{1-\frac{4}{\gamma}\left[\frac{1}{2}+\frac{\sin (\pi \alpha)}{\pi}+\left(\frac{1}{2}-\alpha\right) \cos (\pi \alpha)\right]\right\} \text {. }
$$

The TLL is maintained under a crossover temperature at which the linear temperature-dependent entropy breaks down; see Fig. 2. The exact analytic expression of thermodynamic functions (22) from the usual TLL description is only accurate for a limited range of temperatures and density. However, the TLL description is incapable of describing quantum criticality for it does not contain the right fluctuations in the critical regime. The equation of state (20) contains the proper universal scaling functions, which control the full thermodynamical properties in the quantum critical regimes. Near the critical point, the thermal dynamical properties can be cast into universal scaling forms, for example, (16) and (17). There exist no-longer-free fermions in the $\mathrm{TLL}_{F}$ phase, shown in Fig. 2, due to the presence of an exponentially small number of bosons at finite temperatures. They behave like a TLL for temperatures below the crossover temperatures (white triangles and squares). The $\mathrm{TLL}_{M}$ stands for a two-component TLL of the mixture of bosons and fermions described by (22).

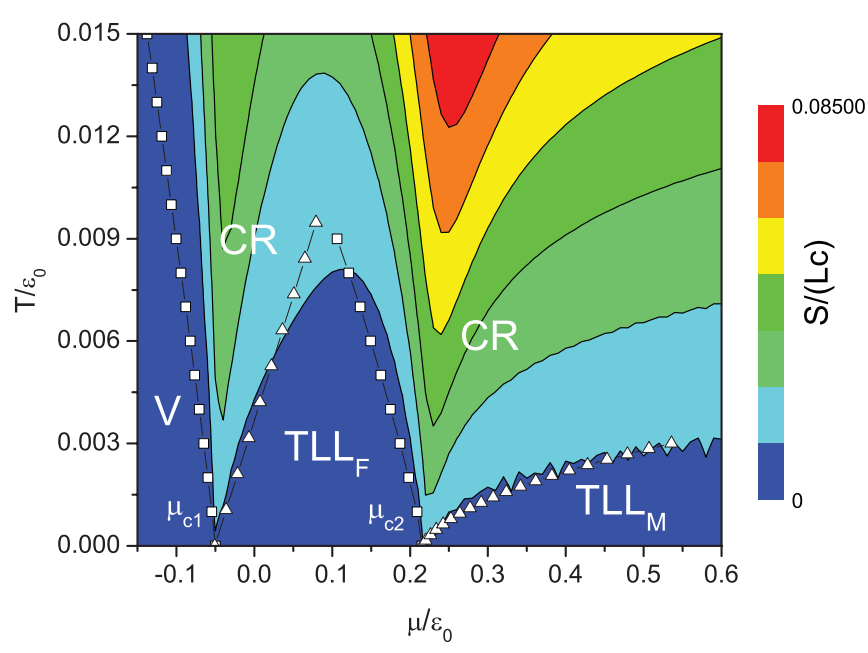

FIG. 2. (Color online) Contour plot of entropy $S$ vs chemical potential from the TBA (3): quantum criticality driven by the chemical potential for $H=0.1 \varepsilon_{0}$. The crossover temperatures (white squares and triangles) separating vacuum, $\mathrm{TLL}_{F}$, and $\mathrm{TLL}_{M}$ from the quantum critical regimes are determined from the breakdown of linear temperature-dependent entropy from (22). Here the TLL $F$ stands for the TLL of fermions where an exponentially small number of bosons are populated at finite temperatures, whereas $\mathrm{TLL}_{M}$ denotes the TLL of the mixture. The vacuum evolves into a quasiclassical regime at finite temperature. 

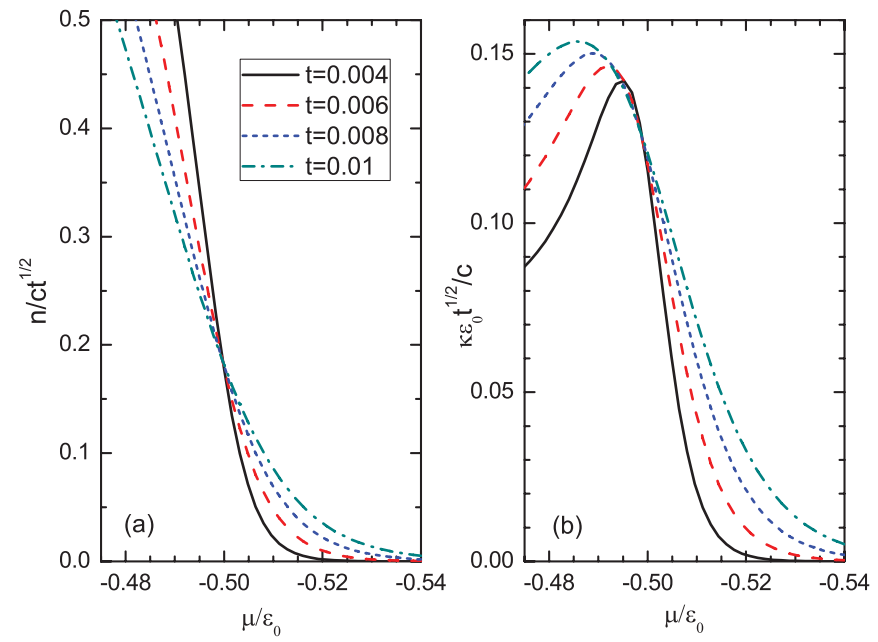

FIG. 3. (Color online) The density $n_{b}$ and compressibility $\kappa_{b}$ vs chemical potential $\mu$ for $H / \varepsilon_{0}=-1$ at different temperatures. The curves intersect at the critical point $\mu=H / 2$, i.e., the phase boundary between the pure boson phase and the vacuum. Here $t=T / \varepsilon_{0}$.

It is straightforward to work out the critical exponents for the phase transition from vacuum into the free fermions and into the Bose gas phase, i.e., $d=1, z=2$, and $v=1 / 2$; see $[22,24,25]$. Using the TBA equations (3), we verify the scaling behavior of the mixture for the phase transitions from vacuum into the pure Bose state, shown in Fig. 3, and from vacuum into the pure Fermi state, shown in Fig. 4. We see that both the density and compressibility at different temperatures intersect at the critical point. For both cases, the compressibility always evolves a round peak as the temperature decreases. This indicates the density of state changes when the phase transition occurs. As the temperature approaches the limit $T \rightarrow 0$, the compressibility tends to divergence.

Comparing the density, which is numerically obtained from the pressure (4), with the universal scaling form of Eq. (16),

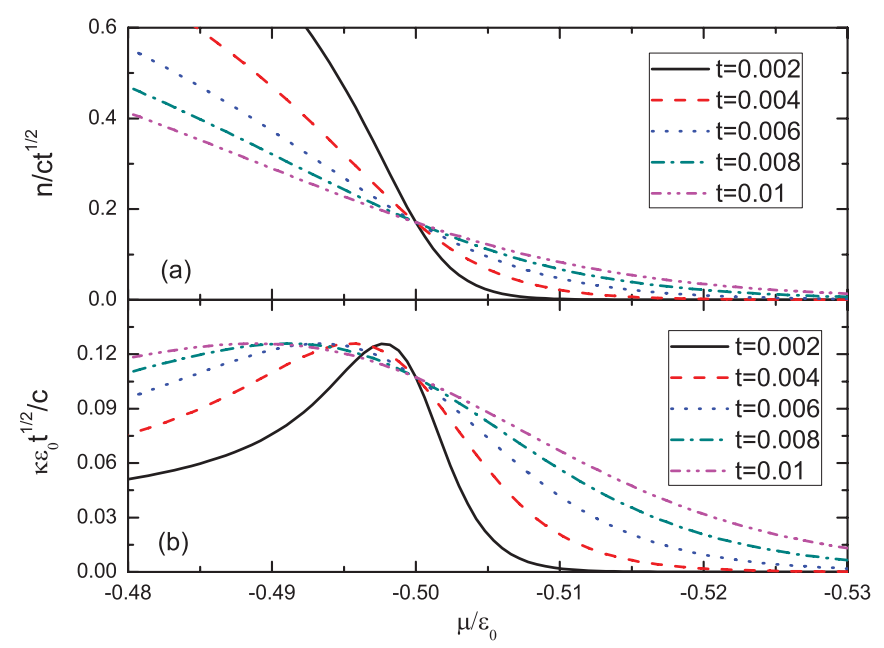

FIG. 4. (Color online) The density $n_{f}$ and compressibility $\kappa_{f}$ vs chemical potential $\mu$ for $H / \varepsilon_{0}=1$ at different temperatures. The curves intersect at the critical point $\mu=-H / 2$, i.e., the phase boundary between the pure fermion phase and the vacuum. Here $t=T / \varepsilon_{0}$.
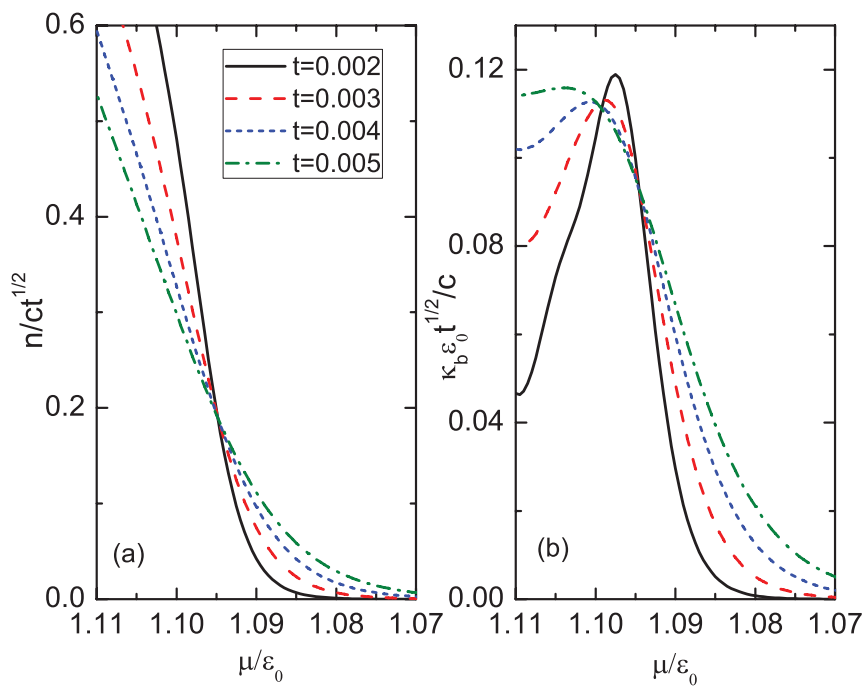

FIG. 5. (Color online) The density $n_{b}$ and compressibility $\kappa_{b}$ vs chemical potential $\mu$ for $H / \varepsilon_{0}=1$ at different temperatures. In this setting, the intersection nature can map out the zero-temperature phase boundary for the phase transition from the free fermions into the mixture of bosons and fermions. Here $t=T / \varepsilon_{0}$.

we can extract the critical exponent $z=2$ and the correlation length exponent $v=1 / 2$ with the dimensionality $d=1$. Here we numerically demonstrate the universal scaling behavior of the mixture of bosons and fermions in Figs. 3-5. For practical convenience, here we have chosen the density of bosons $n_{b}$ to demonstrate the intersections for the phase transitions from vacuum into the phase of pure bosons, and from the phase of fermions into the mixture, where $n_{b}$ does not have a background near the transition points; see Figs. 3 and 5. For a fixed effective magnetic field $H$, we see that by a proper temperature scaling, the density curves $n_{b}(T) / \sqrt{T}$ at different temperatures intersect at the points $\mu_{c}$. In Fig. 5, we display the scaled density distributions for different temperatures by numerically solving the TBA equations (3)-(5). It is also seen that the compressibility curves at different temperatures intersect at the critical point $\mu_{c}$. The compressibility tends to divergence as the temperature tends to zero. It evolves to a round peak at low temperature due to the change of the density of states around the critical points. The quantum criticality near the critical points associating the phase transitions from vacuum into the phase of pure bosons and the phase of pure fermions reveals a subtle difference in thermodynamical properties; see Figs. 3 and 5.

\section{QUANTUM CRITICALITY IN THE HARMONIC TRAP}

In the experiment with cold atoms, the 1D quantum gas is realized by tightly confining the atomic cloud in two (radial) dimensions and weakly confining it along the axial direction in an external harmonic trap. For the mixture of bosons and fermions in a harmonic trap, we can calculate its density distribution profiles by evaluating the thermodynamical dynamics within the local-density approximation. According to the local-density approximation, the system reaches local equilibrium in each small interval around each point $x$ in the external trap. The density distribution of the trapped gas is 
then obtained via the local equation of state [16,34]. Within the local-density approximation, the chemical potentials in the equation of state (4) as well as in the TBA equations are replaced by the local chemical potentials given by

$$
\begin{aligned}
& \mu_{b}(x)=\mu_{b}(0)-V_{b}(x), \\
& \mu_{f}(x)=\mu_{f}(0)-V_{f}(x) .
\end{aligned}
$$

Here the external potential is defined as $V_{b}(x)=V_{f}(x)=$ $m \omega^{2} x^{2} / 2$ with harmonic frequency $\omega$, and the characteristic length for the harmonic trap is $a=\sqrt{\hbar / m \omega}$. For this case, Eqs. (25) and (26) can be alternatively represented as

$$
\mu(y) / \varepsilon_{0}=\mu(0) / \varepsilon_{0}-y^{2}
$$

for a fixed $H$, where the dimensionless coordinate is given by $y=x /\left(a^{2} c\right)$. From the Bethe ansatz equations, the dimensionless density $n_{b} / c$ and $n_{f} / c$ can be obtained for fixed dimensionless chemical potential $\mu_{b} / \varepsilon_{0}$ and $\mu_{f} / \varepsilon_{0}$. The total particle number $N$ is obtained from

$$
\frac{N a_{1 \mathrm{D}}^{2}}{a^{2}}=4 \int_{-\infty}^{\infty} \frac{n_{b}(y)+n_{f}(y)}{c} d y
$$

with the $1 \mathrm{D}$ scattering length $a_{1 \mathrm{D}}=-2 / c$. We define a polarization rate between the Bose particle number and the total particle number $\alpha \equiv N_{b} / N$. For fixed $\mu(0) / \varepsilon_{0}$ and $H / \varepsilon_{0}$, we can calculate $N a_{1 \mathrm{D}}^{2} / a^{2}$ and $\alpha$. In the presence of the confined potential, the length scale of the system at quantum criticality is still much smaller than the trap size. Therefore, the critical behavior of the homogeneous gas can be mapped out by the density profiles of gas at finite temperatures [22].

We fix $N a_{1 \mathrm{D}}^{2} / a^{2}$ and $\alpha$ in the trapped gas of the mixture; the density profiles reveal a universal scaling behavior of the gas. Figure 6 shows the density profiles of bosons and fermions in the harmonic trap for $N a_{1 \mathrm{D}}^{2} / a^{2}=1$ and $\alpha=N_{b} / N=0.5$ at different temperatures. Here we find that bosons and fermions coexist in the trap center accompanied by the phase of pure fermions at the edges. We further demonstrate how to map out the zero-temperature phase boundaries from the density profiles of the trapped gas at finite temperatures. In Figs. 7(a)

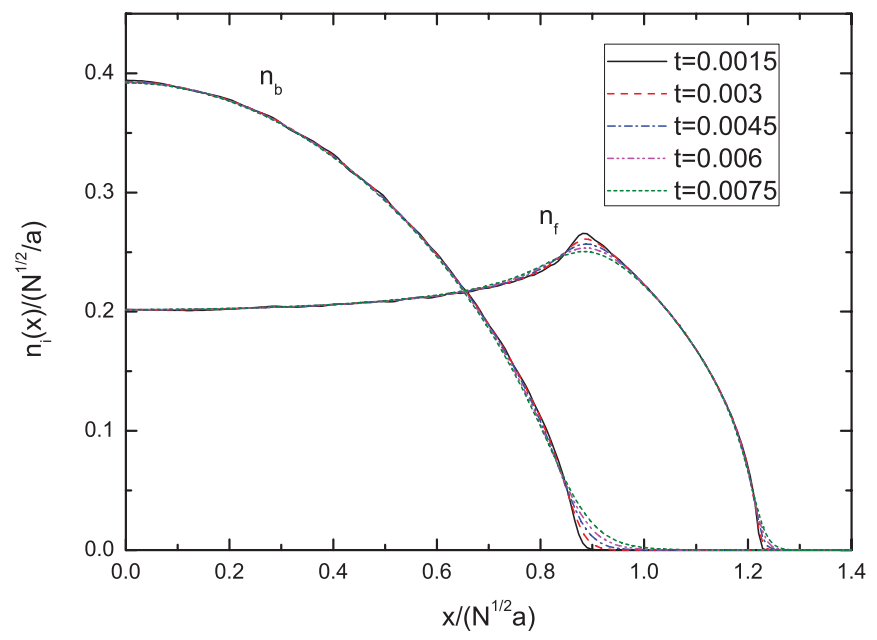

FIG. 6. (Color online) The density distribution at different temperatures with $N^{t} a_{1 \mathrm{D}}^{2} / a^{2}=1$ and $\alpha=0.5$.

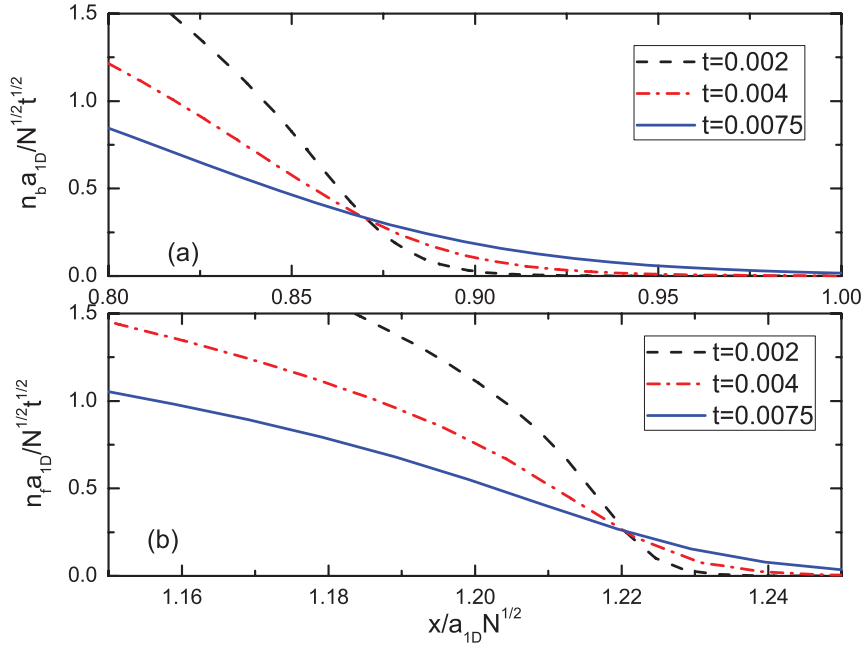

FIG. 7. (Color online) The densities $n_{b}$ and $n_{f}$ vs normalized position for $N a_{1 \mathrm{D}}^{2} / a^{2}=1$ and $\alpha=0.5$ at different temperatures. The density curves intersect at the critical point that maps out the phase boundaries of the trapped gas. Here $t=T / \varepsilon_{0}$.

and 7(b), we demonstrate the scaled density distributions of bosons and fermions. It is clearly seen that the scaled density curves for different temperatures intersect at the critical point of the trapped gas. Thus the critical point, separating the mixture of bosons and fermions from the phase of pure fermions, is mapped out. Similarly, the density curves of fermions intersect at the critical point that maps out the phase boundary for the phase transition from vacuum into the phase of free fermions.

\section{CONCLUSION}

In summary, we have studied the phase diagram, universal TLL, and quantum criticality of the 1D Bose-Fermi mixture by means of the TBA equations. We have derived the equation of state and universal TLL thermodynamics of the model for strong repulsion. We have proven that the low-energy physics of the Bose-Fermi mixture are described by a two-component TLL. The universal scaling behavior of thermodynamical properties at quantum criticality provides a physical origin of quantum critical phenomena. Furthermore, the quantum criticality of the Bose-Fermi mixture in a harmonic trap has been studied within the local-density approximation. It turns out that the phase diagram and critical properties of the bulk system can be mapped out from the density profiles of the trapped mixture gas at finite temperatures. Our exact results can help with the experimental study of quantum critical phenomena in a 1D harmonic trap.

\section{ACKNOWLEDGMENTS}

This work was supported by the National Program (Grant No. 2011CB921700) Basic Research of MOST, NSF of China under Grants No. 10821403, No. 11174360, and No. 10974234, and the 973 Program grant. The work of X.-W.G. has been partially supported by the Australian Research Council. Y.Z. is also supported by the 973 Program under Grant No. 2011CB921601, and the Program for NCET. 
[1] F. Schreck, L. Khaykovich, K. L. Corwin, G. Ferrari, T. Bourdel, J. Cubizolles, and C. Salomon, Science 291, 2570 (2001); Phys. Rev. Lett. 87, 080403 (2001).

[2] Z. Hadzibabic, C. A. Stan, K. Dieckmann, S. Gupta, M. W. Zwierlein, A. Gorlitz, and W. Ketterle, Phys. Rev. Lett. 88, 160401 (2002); G. Roati, F. Riboli, G. Modugno, and M. Inguscio, ibid. 89, 150403 (2002); S. Inouye, J. Goldwin, M. L. Olsen, C. Ticknor, J. L. Bohn, and D. S. Jin, ibid. 93, 183201 (2004).

[3] T. Fukuhara, S. Sugawa, Y. Takasu, and Y. Takahashi, Phys. Rev. A 79, 021601(R) (2009).

[4] M. Olshanii, Phys. Rev. Lett. 81, 938 (1998).

[5] A. Görlitz et al., Phys. Rev. Lett. 87, 130402 (2001).

[6] H. Moritz, T. Stöferle, M. Köhl, and T. Esslinger, Phys. Rev. Lett. 91, 250402 (2003); T. Stöferle, H. Moritz, C. Schori, M. Köhl, and T. Esslinger, ibid. 92, 130403 (2004).

[7] B. Paredes, A. Widera, V. Murg, O. Mandel, S. Fölling, I. Cirac, G. V. Shlyapnikov, T. W. Hänsch, and I. Bloch, Nature (London) 429, 277 (2004).

[8] T. Kinoshita, T. Wenger, and D. S. Weiss, Science 305, 1125 (2004).

[9] E. Haller, M. Gustavsson, M. J. Mark, J. G. Danzl, R. Hart, G. Pupillo, and H.-C. Nägerl, Science 325, 1224 (2009).

[10] A. H. van Amerongen, J. J. P. van Es, P. Wicke, K. V. Kheruntsyan, and N. J. vanDruten, Phys. Rev. Lett. 100, 090402 (2008).

[11] Y. Liao, A. S. C. Rittner, T. Paprotta, W. Li, G. B. Partridge, R. G. Hulet, S. K. Baur, and E. J. Mueller, Nature (London) 467, 567 (2010).

[12] K. K. Das, Phys. Rev. Lett. 90, 170403 (2003).

[13] M. A. Cazalilla and A. F. Ho, Phys. Rev. Lett. 91, 150403 (2003).

[14] L. Mathey, D. W. Wang, W. Hofstetter, M. D. Lukin, and E. Demler, Phys. Rev. Lett. 93, 120404 (2004).

[15] C. K. Lai and C. N. Yang, Phys. Rev. A 3, 393 (1973); C. K. Lai, J. Math. Phys. 15, 954 (1974).
[16] A. Imambekov and E. Demler, Phys. Rev. A 73, 021602 (2006); Ann. Phys. 321, 2390 (2006).

[17] H. Frahm and G. Palacios, Phys. Rev. A 72, 061604 (2005).

[18] M. T. Batchelor, M. Bortz, X. W. Guan, and N. Oelkers, Phys. Rev. A 72, 061603 (2005); X.-W. Guan, M. T. Batchelor, and J.-Y. Lee, ibid. 78, 023621 (2008).

[19] X. G. Yin, S. Chen, and Y. B. Zhang, Phys. Rev. A 79, 053604 (2009).

[20] M. P. A. Fisher, P. B. Weichman, G. Grinstein, and D. S. Fisher, Phys. Rev. B 40, 546 (1989).

[21] S. Sachdev, Quantum Phase Transitions (Cambridge University Press, Cambridge, 1999).

[22] Q. Zhou and T.-L. Ho, Phys. Rev. Lett. 105, 245702 (2010).

[23] X. Cai, S. Chen, and Y. Wang, Phys. Rev. A 83, 043613 (2011).

[24] X. G. Yin, X.-W. Guan, S. Chen, and M. T. Batchelor, Phys. Rev. A 84, 011602(R) (2011); X.-W. Guan and T.-L. Ho, ibid. 84, 023616 (2011).

[25] X.-W. Guan and M. T. Batchelor, J. Phys. A 44, 102001 (2011).

[26] K. R. A. Hazzard and E. J. Mueller, Phys. Rev. A 84, 013604 (2011); S. Fang, C.-M. Chung, P. N. Ma, P. Chen, and D.-W. Wang, ibid. 83, 031605(R) (2011).

[27] Tin-Lun Ho and Qi Zhou, Nature Phys. 6, 131 (2010).

[28] N. Gemelke, X. Zhang, C.-L. Hung, and C. Chin, Nature (London) 460, 995 (2009).

[29] W. S. Bakr, A. Peng, M. E. Tai, R. Ma, J. Simon, J. I. Gillen, S. Föling, L. Pollet, and M. Greiner, Science 329, 547 (2010).

[30] J. F. Sherson, C. Weitenberg, M. Endres, M. Cheneau, I. Bloch, and S. Kuhr, Nature (London) 467, 68 (2010).

[31] C.-L. Huang et al., Nature (London) 470, 238 (2011).

[32] C. N. Yang and C. P. Yang, J. Math. Phys. 10, 1115 (1969).

[33] Z.-X. Hu, Q.-L. Zhang, and Y.-Q. Li, J. Phys. A 39, 351 (2006).

[34] V. Dunjko, V. Lorent, and M. Olshanii, Phys. Rev. Lett. 86, 5413 (2001); C. Menotti and S. Stringari, Phys. Rev. A 66, 043610 (2002).

[35] R. Coldea et al., Science 327, 177 (2010). 